\title{
DESIGN PROCEDURE TO OPTIMIZE THE PERFORMANCE OF A TIDAL CURRENT TURBINE BLADE
}

\author{
CHUL-HEE JO, KANG-HEE LEE \& SU-JIN HWANG \\ Inha University, South Korea
}

\begin{abstract}
The key element for converting in-line flow energy into rotational motion is a tidal turbine, which is one of the crucial components directly affecting the performance and efficiency of a tidal energy convertor. The tidal turbine is designed using blade element momentum theory as it has large similarities to the wind turbine. However, differences exist in the range of Reynolds number and fluid properties that should be considered for tidal turbine design. In this study, an airfoil was selected for the section of the tidal blade, and the 2D performance was evaluated by a verified computational fluid dynamics (CFD) method to optimize the blade shape. Based on this procedure, a horizontal-axis tidal turbine of $10 \mathrm{~m}$ diameter was designed considering the operating conditions. The performance of the designed turbine has been evaluated using three-dimensional CFD analysis and compared and validated with the experiment using a $1 / 20$ scaled model in the circulating water channel to optimize the performance of the turbine in water.
\end{abstract}

Keywords: tidal current power, horizontal axis turbine, blade element momentum theory (BEMT), tidal energy convertor (TEC).

\section{INTRODUCTION}

A tidal blade can generate lift and drag forces together with torque and thrust from rotation caused by incoming flow. The characteristic affecting the efficiency of the blade can be determined using the blade element momentum theory (BEMT). Previously, quite a few studies have been introduced about the application of the BEMT to the tidal turbine design [1], [2] and the actuator disk theory was also presented, which can simulate the flow field around the simplified turbine [3]. Most airfoils have been developed for aircraft or wind turbines, and accurate characteristics were presented from wind tunnel experiments [4]. However, the chord-length-based Reynolds number of the tidal blade is approximately 10 times larger than that of the wind blade. Also, the wind tunnel test conditions are different from that of the ocean. Therefore, most tidal turbine studies have been carried out using numerical codes employing a panel method such as X-Foil to estimate two-dimensional airfoil characteristics in water. In this study, using a validated computational fluid dynamics (CFD) analysis method with experimental data obtained from the flume tank, two-dimensional airfoil performance was evaluated considering the operating conditions of the tidal turbine. Based on the obtained characteristics of the airfoil, the optimized design of a horizontal axis tidal turbine of $10 \mathrm{~m}$ diameter was executed. The performance of the optimized turbine has been evaluated using three-dimensional CFD analysis. The results of the performance evaluation have also been verified using a 1/20 scaled-model test conducted in the circulating water channel (CWC).

\section{SELECTION OF AN AIRFOIL FOR BLADE SECTION}

To design a highly efficient turbine blade, it is important to have a high lift-drag ratio, with a significant difference between the optimal angle of attack and the stall region. The airfoils, including NACA 63-221, NACA 63-421, S809 and S814, have been initially reviewed and investigated to select the suitable blade shape for the tidal turbine. The lift and drag coefficients for the angle of attack for each airfoil were compared. The stall did not occur 
rapidly in NACA 63-221 but the lift coefficient was no bigger than other airfoils, with a low performance in the range of the small angle of attack. NACA 63-421 had a higher lift coefficient than NACA 63-221 and performance was stable over the stall region. The S809 developed by NREL appeared to perform well over a wide range of angles of attack, but the lift coefficient was not as high as that of NACA 63-221. S814 did not show any significant loss, even in the stall region, and generated a high lift coefficient in the range of the low angle of attack. Fig. 1 shows the pressure coefficient distribution with respect to chord length for each airfoil. It was confirmed that the pressure difference was distributed evenly in S814 by generating high lift compared to other airfoils near the trailing edge. The S814 airfoil was selected for the tidal blade by comparing lift and drag coefficients and chord-wise pressure coefficient distribution.

The parameters to be considered for determining the optimal angle of attack are the stalling angle and the lift-drag ratio. An airfoil which has a higher lift-drag ratio is more suitable to the turbine blade as it can improve the turbine performance. The optimal angle of attack that shows a high lift-drag ratio should also be away from the stalling angle for stable operation. The maximum lift-drag ratio of S814 in the water is 81 at a $6^{\circ}$ angle of attack, which is away more than $10^{\circ}$ from the stalling angle (Fig. 2). Therefore, the $6^{\circ}$ angle was determined to be the optimal angle of attack and was applied to the tidal blade design.



(a) NACA 63-221

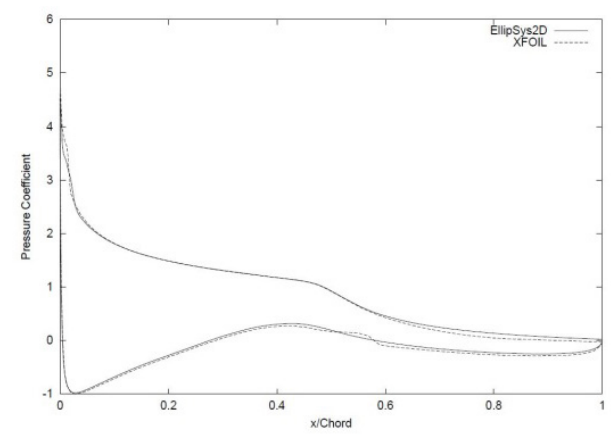

(c) S809



(b) NACA 63-421

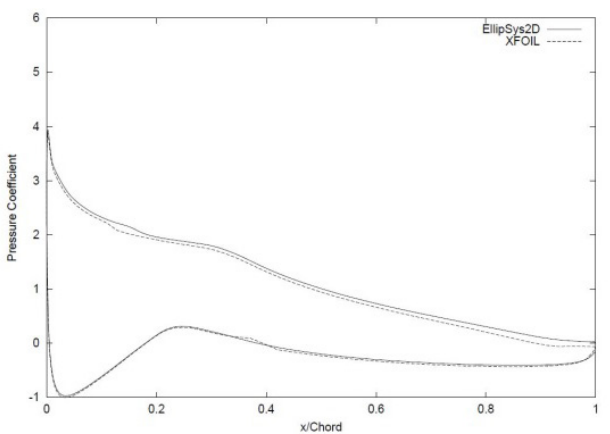

(d) S814

Figure 1: (a)-(d) Pressure coefficient vs. x/Chord [4]. 



Figure 2: Comparison of lift and drag curves.

\section{TIDAL TURBINE DESIGN}

The design velocity and turbine diameter were determined as $2.5 \mathrm{~m} / \mathrm{s}$ and $10 \mathrm{~m}$, respectively, considering the coastal site conditions for tidal device installation in South Korea. The tidal turbine blade was designed using an algorithm, as shown in Fig. 3. The design parameters applied in the study are flow velocity, tip speed ratio (TSR), optimal angle of attack, turbine diameter, number of blades, etc., as summarized in Table 1 . The blade design involves a procedure in which a specific airfoil is arranged with an appropriate twist angle and chord length. $P$ in eqn (1) represents the power that can be extracted from the tidal current which is to be defined as the product of generated power from the turbine and the efficiency of power train $\eta$. The power train coefficient, $\eta$, is generally determined as 0.9. Considering the mechanical loss by friction in a watertight bearing, gearbox, etc., it was conservatively assumed to be 0.85 in the study. The rated power is about $200 \mathrm{~kW}$ with the design TSR $(\lambda)$ of 5 and the rated rotational speed of 24 RPM based on eqns (2) and (3). The chord length $C$ is calculated based on eqn (4). 


$$
\begin{gathered}
P=\eta C_{p}\left(\frac{\rho \pi D^{2} U_{D}^{3}}{8}\right), \\
N_{r p s}=\frac{U_{D}}{\pi D} \lambda, \\
T S R=\frac{U_{t i p}}{U_{\infty}}=\frac{\omega R}{U_{\infty}}, \\
\frac{C}{R}=\frac{2 \pi}{N \lambda C_{L}} \times \frac{4 \lambda \mu^{2} a}{\sqrt{(1-a)^{2}+\left\{\lambda \mu\left(1+a^{\prime}\right)\right\}^{2}}} .
\end{gathered}
$$

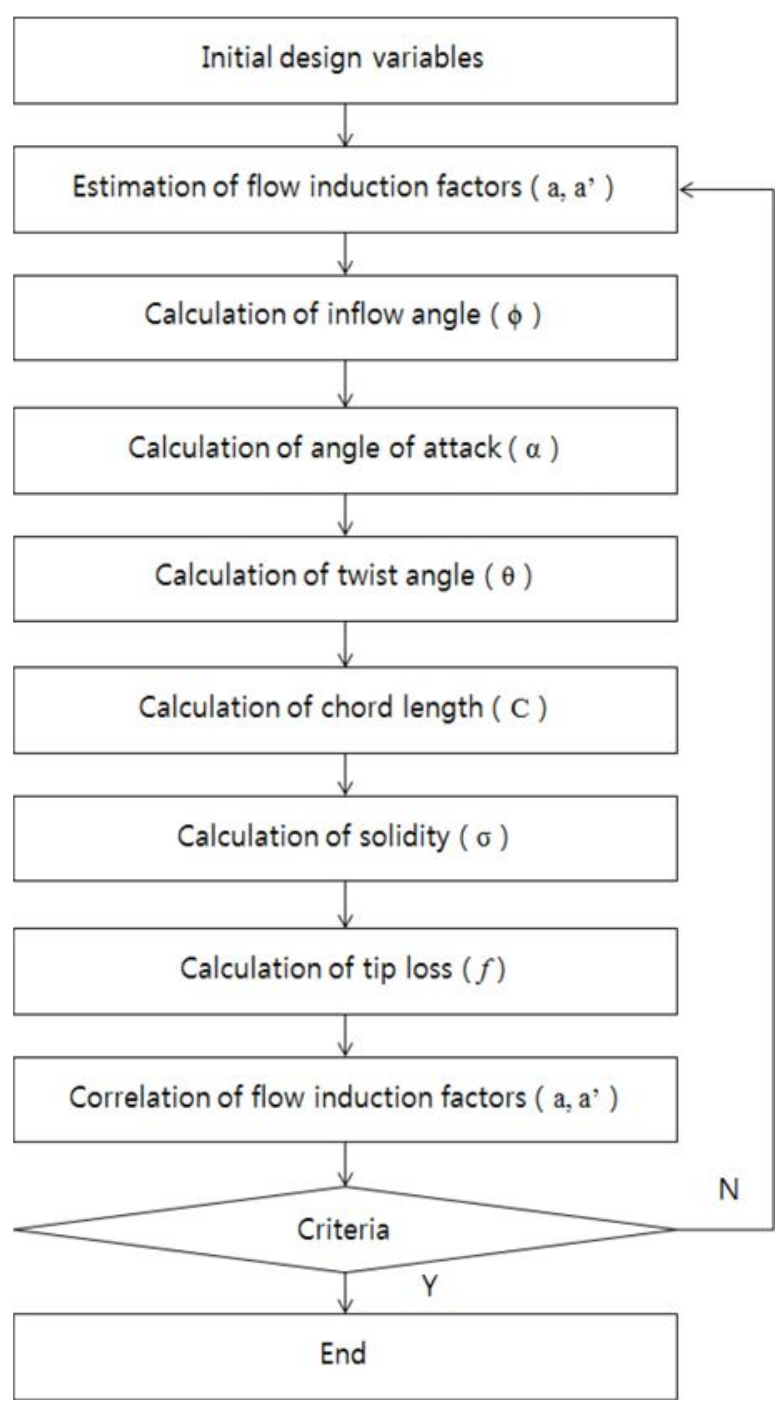

Figure 3: Algorithm for turbine design. 
Table 1: Design parameters of tidal turbine.

\begin{tabular}{|l|l|l|}
\hline \multicolumn{2}{|c|}{ Design parameters } & Values \\
\hline$P$ & Rated power (kW) & 200 \\
\hline$C_{p}$ & Power coefficient & 0.4 \\
\hline$U_{D}$ & Design velocity (m/s) & 2.5 \\
\hline$\Lambda$ & Tip speed ratio & 5 \\
\hline$A$ & Angle of attack (deg) & 6 \\
\hline$D$ & Turbine diameter (m) & 10 \\
\hline$N$ & Blade number (EA) & 3 \\
\hline$\omega$ & Angular speed (rpm) & 24 \\
\hline
\end{tabular}

After estimating flow-induction factors, the twist angle and chord length for each blade section can be obtained using the iteration procedure according to the design algorithm. Every blade section contributing to the turbine's performance should satisfy the optimal angle of attack at operating condition. The optimal angle of attack of $6^{\circ}$ determined by CFD analysis in this study is the most important variable in blade design procedure. To confirm the turbine design, a three-dimensional modelling was performed using CATIA commercial software, as shown in Fig. 4.

ANSYS CFX, a commercial CFD code, was used to analysis the turbine performance. The torque of the blade that is related to the performance of blade was calculated. As the turbine rotates in steady rotating speed with uniform incoming flow, the flow pattern near the blades does not vary significantly as per time, which can be considered as a quasi-steady state for a horizontal-axis turbine. From various steady-state simulations, the performance of the horizontal-axis turbine can be estimated accurately compared to fully unsteady simulation. The analysis field was assumed to be incompressible, three dimensional, and steady-state, with a two-domain composition consisting of one passage with one blade. An internal rotating domain that encompassed the blades and a stationary domain covered the remaining area of the flow field were simulated, as shown in Fig. 5. The computational domains were calculated by assuming that the flow between adjacent passages was periodic in rotating direction. The upstream length and channel radius are $30 \mathrm{~m}$ (3D). A downstream length was set to $60 \mathrm{~m}$ (6D). Grid generation was precisely conducted for smooth convergence and reliable results. A hybrid grid system was applied to the flow field, as shown in Figs 5 and 6. Since the key areas of interest congregate around the blade in the rotating domain, a finer grid was required for a more accurate estimation of the torque on the blade. Therefore, a dense tetra-prism mesh was generated in the subdomain with complex geometry around the blade. The stationary domain was filled with hexahedral mesh.
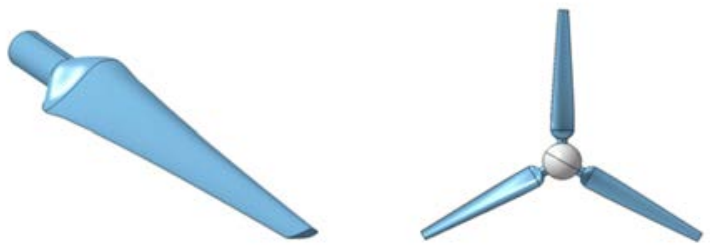

Figure 4: 3D modelling of designed turbine. 


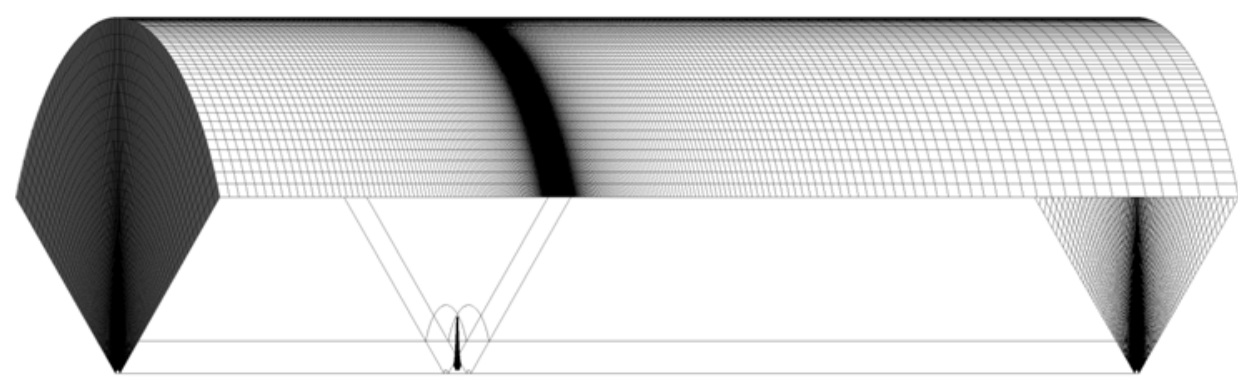

Figure 5: Computational domain with mesh lines.



Figure 6: Hybrid grid system in rotating domain.

The specifications of computational domain and mesh information are described in Table 2. Solid boundaries and blade surfaces were defined as non-slip walls. The hub and top boundary were treated as a free slip wall to eliminate their effect. In the rotating domain, an angular velocity was prescribed for each case having TSR from 1 to 9 using the moving reference frame (MRF) method. The surfaces between two domains were interfaced using the general grid interface (GGI) method, and the frozen rotor method was applied for the MRF interface.

A rotational periodic model was used for the interface between side wall boundaries. A normal velocity was applied to the inlet from $1.0 \mathrm{~m} / \mathrm{s}$ to $3.5 \mathrm{~m} / \mathrm{s}$ without vertical and lateral gradient to eliminate the environmental effects, and the static pressure was set to $1.0 \mathrm{~atm}$ at the outlet for all cases. The shear stress transport (SST) turbulence model was used as a turbulence closure. In the SST model, a k- $\omega$ model at the near-wall, and a k- $\varepsilon$ model were applied beyond the wall region. Table 3 shows the defined boundary conditions. 
Table 2: Domain specification and grid information.

\begin{tabular}{|l|l|}
\hline Description & Specification \\
\hline Blade radius & $5 \mathrm{~m}$ (turbine dia. $=10 \mathrm{~m}$ ) \\
\hline Upstream length & 3D \\
\hline Downstream length & $6 \mathrm{D}$ \\
\hline Radius of flow channel & 3D \\
\hline Total number of nodes & $4,034,067$ \\
\hline Total number of elements & $7,356,988$ \\
\hline
\end{tabular}

Table 3: Boundary conditions.

\begin{tabular}{|l|l|}
\hline Description & Analysis condition \\
\hline Analysis type & Steady state \\
\hline Inlet & Normal speed \\
\hline Outlet & Static pressure (1 atm) \\
\hline Top boundary and hub & Free slip wall \\
\hline Blade & Non-slip wall (smooth surface) \\
\hline Interface area & GGI with frozen rotor \\
\hline Side wall & Rotational periodicity \\
\hline Angular velocity & MRF method \\
\hline Turbulence model & k- $\omega$ SST \\
\hline
\end{tabular}

Torque for each TSR was obtained from CFD analysis and CP curves for various upstream velocities were plotted, as shown in Fig. 7. The maximum efficiency of the turbine was obtained as $47.6 \%$ with the rated rotational speed of $24 \mathrm{RPM}$ at $2.5 \mathrm{~m} / \mathrm{s}$ speed at TSR of 5 . The maximum torque of the turbine was measured as $126 \mathrm{kNm}$ at TSR 4 and a rated velocity of $2.5 \mathrm{~m} / \mathrm{s}$. The relation between the incidence angle, angle of attack and TSR could be verified using streamlines around the airfoil. The performance curves show similar trends for various upstream velocities. It was confirmed that there were no significant changes in turbine efficiency for varying upstream velocities. The turbine is to be operated at the optimal TSR 5 by the maximum power point tracking (MPPT) method. The streamline distribution of the $70 \%$ section of the designed blade is shown in Fig. 8 . The flow incidence angle into the airfoil can be checked for various TSRs. The attached flow should be formed on the suction side to generate high performance. The flow was separated from the leading edge at TSR of 2 that induced large vortex all over the suction side. At TSR 3, the separation occurred from $30 \%$ point of the chord inducing a vortex near the trailing edge. The attached flow was observed on the suction side from TSR 4, and an expected flow-incidence angle was formed at the rated TSR 5 . 




Figure 7: Cp curves for TSR against various upstream velocities.



(a) TSR 2

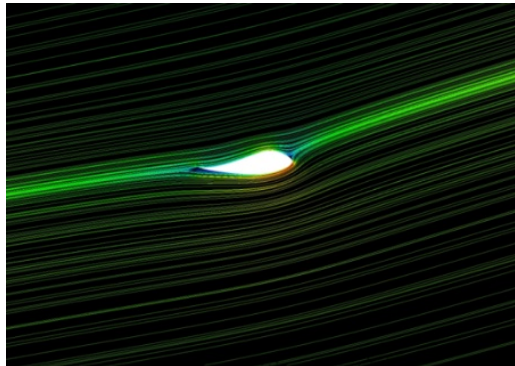

(c) TSR 4



(e) TSR 6



(b) TSR 3



(d) TSR 5

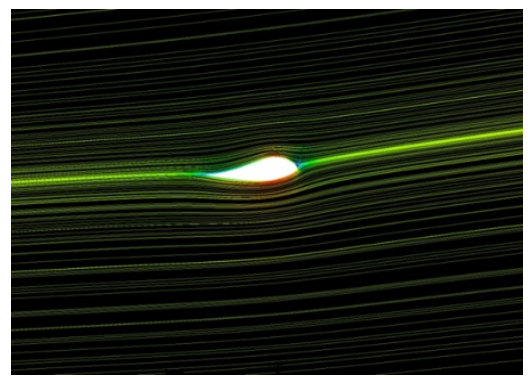

(f) TSR 7

Figure 8: Streamlines around 70\% blade section for various TSRs (a)-(f). 
To verify the turbine performance estimated by CFD, the experiment was conducted with a 1/20 scaled physical model in the CWC in Inha University. The length of the channel is $6.0 \mathrm{~m}$ with a measuring section that was $2.3 \mathrm{~m}$ long, $1.0 \mathrm{~m}$ wide and $0.9 \mathrm{~m}$ deep. A $0.5 \mathrm{~m}$ diameter turbine model was tested with sensors and generator in nacelle which can control the load on the powertrain, as shown in Fig. 9. The rotational speed of the turbine was controlled by the variable electrical load through the generator, and torque was measured with RPM concurrently. The Cp curve obtained from the experiment was plotted in Fig. 10 with results from the CFD analysis.

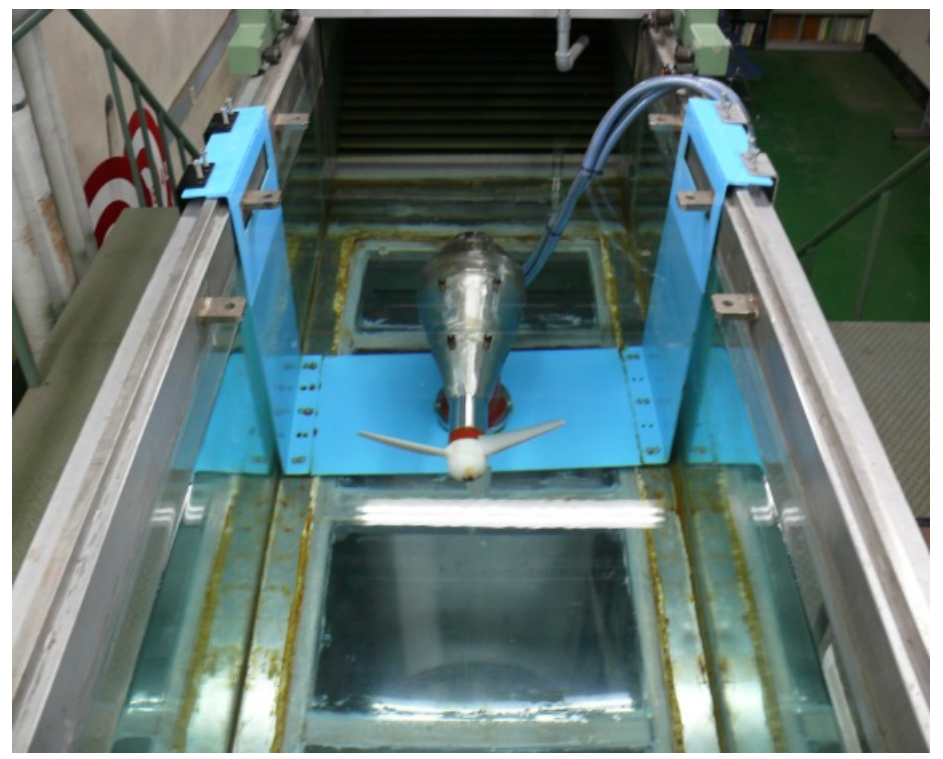

Figure 9: Experiment arrangement in CWC.

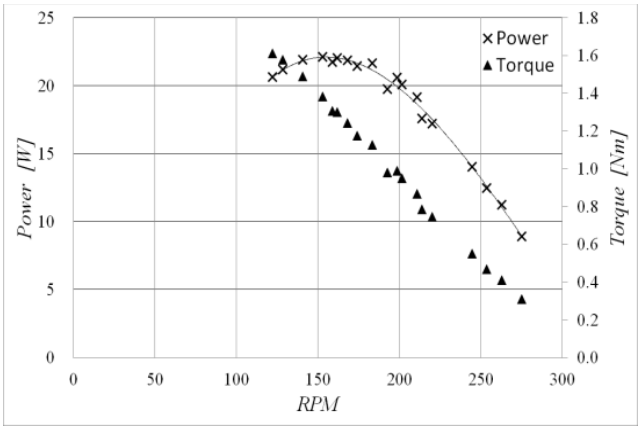

(a) Measured power and torque

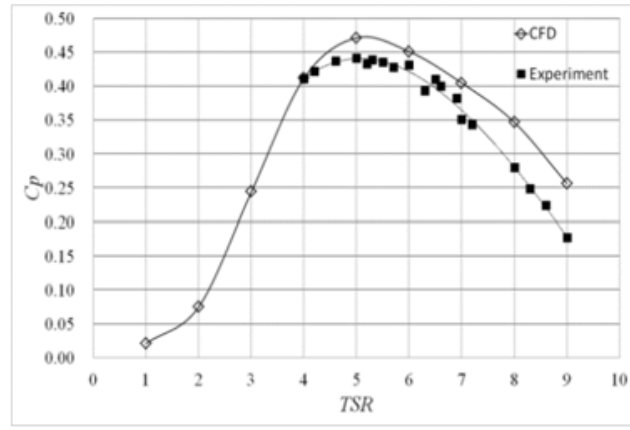

(b) Cp curves

Figure 10: (a) Results of experiment; (b) Comparison of the Cp curves with CFD. 
The turbine speed controlled at 275 RPM, which is idling without a load to $122 \mathrm{RPM}$, was corresponded to TSR 4 by applying the electrical load. The turbine did not rotate under TSR 4, meaning that the maximum torque of turbine was generated at TSR 4, which was consistent with the CFD analysis results. A maximum power of $22 \mathrm{~W}$ was generated at $150 \mathrm{RPM}$ corresponding to TSR 5. The experiment results showed a little lower performance but the Cp curves obtained from experiment and CFD showed good agreement. It is considered that a friction induced by seals at the shaft and mechanical loss caused the discrepancy. It was found that a high-performance tidal turbine design can be optimized with airfoils by analysing the airfoil characteristics in the water. The results of CFD analysis for performance evaluation have also been verified in the experiment.

\section{CONCLUSION}

As there are significant differences in operating environments, especially in Reynolds numbers and working fluids, the characteristics of airfoils developed for wind turbines should be verified in order to be applied to a tidal turbine. The numerical results showed different characteristics in lift and drag coefficients of the S814 compared to experimental data obtained from the wind tunnel - as the angle of attack increased, bigger discrepancies were found. Based on the characteristics of S814, the horizontal-axis tidal turbine of $10 \mathrm{~m}$ diameter was designed using BEMT. The turbine performance was evaluated by both CFD analysis and the experiment, and the $\mathrm{Cp}$ curves showed good agreement. From the procedure introduced in this research, the tidal turbine design can be optimized to obtain the maximum efficiency and performance.

\section{ACKNOWLEDGEMENTS}

This research was a part of the project titled 'Manpower training program for ocean energy', funded by the Ministry of Oceans and Fisheries, Korea and also supported by the Korea Institute of Energy Technology Evaluation and Planning (KETEP) and the Ministry of Trade, Industry \& Energy (MOTIE) of the Republic of Korea (no. 20163030071850).

\section{REFERENCES}

[1] Batten, W.M.J., Bahaj, A.S., Molland, A.F. \& Chaplin, J.R., Hydrodynamics of marine current turbines. Journal of Renewable Energy, 31(2), pp. 249-256, 2006.

[2] Baltazar, J. \& Campos, J., Hydrodynamic analysis of horizontal axis marine current turbine with a boundary element method. Proc. of International Conference on Ocean, Offshore and Arctic Engineering, 2008.

[3] Harrison, M.E., Batten, W.M.J. \& Bahaj, A.S., A blade element actuator disc approach applied to tidal stream turbines. Proc. of Oceans '10, 2010.

[4] Bertagnolio, F., Sorensen, N., Johansen, J. \& Fuglsang, P., Wind turbine airfoil catalogue, Riso National Laboratory, Roskilde, Denmark. 2001. 